\title{
Fluxo de informações no mercado brasileiro de alimentos: A influência da crise
}

\section{financeira de 2008}

\author{
Information flow in brazilian food market: The influence of the 2008 financial crisis
}

Flujo de información en el mercado brasileño de alimentos: La influencia de la crisis financiera de

Thiago Matheus Paiva do Passo

ORCID: https://orcid.org/0000-0002-4569-374X Universidade Federal Rural de Pernambuco, Brasil E-mail:thiagompasso@gmail.com

Joelma Mayara da Silva

ORCID: https://orcid.org/0000-0003-2354-6068 Universidade Federal Rural de Pernambuco, Brasil E-mail: jms.estat@gmail.com

Lidiane da Silva Araújo

ORCID: https://orcid.org/0000-0003-3111-7925 Universidade Federal Rural de Pernambuco, Brasil E-mail: lidiane_sac@hotmail.com

Tatijana Stosic

ORCID: https://orcid.org/0000-0002-5691-945X Universidade Federal Rural de Pernambuco, Brasil E-mail: tastosic@gmail.com

\begin{abstract}
Resumo
O agronegócio brasileiro vem ganhando cada vez mais destaque internacional graças à relevante produção e exportação de commodities agrícolas, bem como ao grande potencial de desenvolvimento que esse setor econômico possui no País. $\mathrm{O}$ assunto tem atraído a atenção de diversos pesquisadores porque, à medida que o setor de exportação cresce, tendem a subir os preços dessas commodities para o consumo interno, afetando assim o preço de outros alimentos. Entender a dinâmica da relação de preços entre esses produtos é, portanto, de grande valia para se evitar que possíveis crises financeiras levem também a crises alimentares como a de 2008. Visando contribuir nesse sentido, analisou-se aqui o comportamento dos preços da soja, açúcar, café e boi gordo, por serem commodities agrícolas produzidas em larga escala e comercializadas mundialmente. $\mathrm{O}$ estudo teve como base os preços registrados entre 01/1997 e 12/2019, no CEPEA/ESALQ/USP, considerando os períodos anterior (07/1997 - 06/2007) e o posterior (07/2007 - 12/2019) à crise de 2008. A técnica de análise utilizada foi a Entropia de Transferência (Transfer Entropy TE), que serve para quantificar o fluxo de informação entre pares de séries temporais, bem como para obter a direção desse fluxo. A técnica foi, então, aplicada para cada par de séries de preços considerando os períodos pré-crise e póscrise. Resultados mostraram que a crise afetou a direção de transferência de informação entre os pares açúcar-boi, açúcar-soja e boi-soja. A série de preços do café, por sua vez, transmitiu informação para as outras commodities em ambos os períodos.
\end{abstract}

Palavras-chave: Commodities agrícolas; Entropia de transferência; Crise alimentar.

\begin{abstract}
Brazilian agribusiness is gaining more and more international prominence thanks to the relevant production and export of agricultural commodities, as well as the great potential for development that this economic sector has in the country. The subject has attracted the attention of several researchers because, as the export sector grows, prices of these commodities for domestic consumption tend to rise, affecting the price of other foods. Understanding the dynamics of the price relation between these products is, therefore, of great value in order to avoid that possible financial crises also lead to food crises such as that of 2008. In order to contribute in this sense, the prices behavior of soybean, sugar, coffee and live cattle were analyzed here, as they are agricultural commodities produced on a large scale and traded worldwide. The study was based on the prices registered between 01/1997 and 12/2019, at CEPEA/ESALQ/USP, considering the periods before (07/1997 - 06/2007) and after (07/2007 - 12/2019) the 2008 crisis. The technique of analysis used was the Transfer Entropy - TE, that serves to quantify the flow of information between pairs of time series, as well as to obtain the direction of that flow. The technique was then applied to each pair of time series considering the pre-crisis and post-crisis periods. Results showed that the crisis affected the direction of information transfer between the pairs sugar-cattle, sugar-soybean and cattle-soybean. The time series prices of coffee, in turn, transmitted information to the other commodities in both periods.
\end{abstract}


Keywords: Agricultural commodities; Transfer entropy; Food crisis.

\section{Resumen}

La agroindustria brasileña está ganando cada vez más prominencia internacional gracias a la relevante producción y exportación de commodities agrícolas, así como al gran potencial de desarrollo que tiene este sector económico en el país. El tema ha atraído la atención de varios investigadores porque, a medida que crece el sector de exportación, los precios de estos productos básicos para el consumo interno tienden a subir, lo que afecta el precio de otros alimentos. Comprender la dinámica de la relación de precios entre estos productos es, por tanto, de gran valor para evitar que posibles crisis financieras lleven también a crisis alimentarias como la de 2008. Para contribuir en este sentido, fueron analizados aquí el comportamiento de los precios de soja, azúcar, café y buey gordo, que son commodities agrícolas producidas a gran escala y comercializados mundialmente. El estudio se basó en los precios registrados entre 01/1997 y 12/2019, en CEPEA/ESALQ/USP, considerando los períodos antes (07/1997 - 06/2007) y después (07/2007 12/2019) de la crisis de 2008. Se utilizó la técnica Transfer Entropy, que sirve para cuantificar el flujo de información entre pares de series temporales e obtener la dirección de ese flujo. La técnica se aplicó a cada par de series considerando los períodos anterior y posterior a la crisis. Resultados mostraron que la crisis afectó la dirección de transferencia de información entre los pares azúcar-buey, azúcar-soja y buey-soja. La serie de precios del café, a su vez, transmitió información a las demás commodities en ambos períodos.

Palabras clave: Commodities agrícolas; Entropía de Transferencia; Crisis alimentaria.

\section{Introdução}

A agricultura brasileira vem sofrendo grandes transformações na última década, modernizando-se com novas tecnologias e insumos avançados. Desde o ano de 2010, como exportador agrícola, fica atrás apenas dos Estados Unidos e da União Europeia, mas possui grande potencial de desenvolvimento. Condições favoráveis como solo, clima, além da mecanização do campo e expansão da fronteira agrícola colaboram para a produção de uma agricultura diversificada, destacando-se a soja, o açúcar e o café. No que diz respeito à carne bovina, o fator que propicia o aumento na produção dessa commodity corresponde ao incremento sucessivo da produtividade (Caliari, 2019; Bojanic, 2017).

O Brasil vem assumindo o posto de grande potência no mercado internacional em relação à produção e exportação de commodities (Lima et al., 2019). Esse mercado causa importante impacto econômico no comércio e provoca uma relação de dependência dos preços estabelecidos internacionalmente. Com o aumento da demanda internacional, os preços se elevam e geram lucros para os produtores. Entretanto, num quadro de recessão, ocorre a desvalorização das commodities, comprometendo os lucros das empresas. Verifica-se nesse contexto uma padronização reconhecida internacionalmente. A integração do Brasil a esse mercado torna mais eficaz o mecanismo de transmissão de preços das commodities.

O comércio de commodities agrícolas tem despertado grande cautela na área econômica em razão do aumento dos preços das commodities de alimentos ocorrido na última década (Nazlioglu \& Soytas, 2012; Beckmann \& Czudaj, 2014). A alta do dólar está diretamente relacionada a esse aumento porque causa alterações nos custos dos alimentos importados. A soja, por exemplo, tem seu valor definido em dólar no mercado internacional. Assim, quando o dólar sobe, esse produto também fica mais caro no mercado interno, afetando o preço de outros alimentos.

Um fato marcante para a economia mundial e cujos efeitos despertaram o interesse da comunidade científica foi a crise financeira de 2008. Ela teve como consequência o aumento dos preços de commodities alimentares, ficando por isso também conhecida como a crise alimentar (food crises) de 2008, que resultou na inseguraça alimentar em países em desenvolvimento (Ivanic, Martin, \& Zaman, 2012). Efeitos dessa crise nos preços de commodities agrí́colas brasileiras foram recentemente investigados. Pessoa et al. (2021) identificaram alterações nas correlações entre séries de retorno dos preços de milho, soja e da carne de frango quando comparados os resultados dos períodos anterior e posterior à crise. Farias et al. (2020) observaram que, depois da crise, houve uma redução da entropia das flutuações de preços das commodities açúcar, algodão, arroz, café, milho, e trigo, indicando maior regularidade de preços e, consequentemente, menor eficiência de mercado para essas commodities agrícolas nesse período. 
Diante da visível relevância que o mercado das commodities agrícolas tem para a economia do Brasil e dada a importância de prever alguns dos possíveis efeitos causados por crises financeiras mundiais como a de 2008, investiga-se neste trabalho a relação entre os preços de commodities agrícolas produzidas em larga escala e comercializadas em nível mundial. Foram, então, analisados os preços das commodities: açúcar, soja, café e boi gordo, registrados entre os anos de 1997 e 2019, visando à comparação dessas relações nos períodos anterior e posterior à crise mundial de 2008. Para isso, utilizou-se o método Entropia de Transferência (Transfer Entropy - TE) (Schreiber, 2000), que tem origem na Teoria da Informação e serve para quantificar a direção do fluxo de informação entre séries temporais. Ele foi utilizado em estudos de diversos fenômenos como redes cerebrais (Vincente et al., 2011; Ursino et al., 2020), comportamento animal (Orange \& Abaid, 2015; Shaffer \& Abaid, 2020), vento solar (Wing et al., 2016), plasma de fusão (Van Milligen et al., 2014), e finanças (Sensoy et al., 2014; Jale et al., 2019).

\section{Metodologia}

\subsection{Descrição dos dados}

Os dados analisados foram os preços diários das commodities agrícolas brasileiras açúcar, boi gordo, café e soja, registrados entre janeiro de 1997 e dezembro de 2019 pelo Centro de Estudos Avançados em Economia Aplicada / Escola de Agricultura Luiz de Queiroz / Universidade de São Paulo - CEPEA / ESALQ / USP e disponíveis em http://www.cepea.esalq.usp.br/. A moeda de interesse e a unidade de medida utilizada na cotação de cada uma das commodities agrícolas aqui consideradas encontram-se listadas na Tabela 1.

Tabela 1. Nome das commodities agrícolas sob análise, moeda considerada e as respectivas unidades de medida utilizadas nas cotações.

\begin{tabular}{cc}
\hline Commodity agrícola & Moeda/Unidade de medida \\
\hline Boi gordo & $\mathrm{R} \$ /$ arroba \\
Soja & $\mathrm{R} \$ /$ saca de $60 \mathrm{~kg}$ \\
Açúcar & $\mathrm{R} \$ /$ saca de $50 \mathrm{~kg}$ \\
Café & $\mathrm{R} \$ /$ tonelada \\
\hline
\end{tabular}

Fonte: Elaborada pelos autores a partir dos dados do CEPEA (2020).

\subsection{Entropia de transferência (Transfer Entropy - TE)}

Thomas Schreiber (2000) introduziu uma medida da quantidade de fluxo de informação entre duas séries temporais denominada Entropia de transferência (Transfer Entropy - TE). Essa medida é assim calculada: supondo que $X_{i}$ e $Y_{i}$ sejam variáveis aleatórias discretas, pode-se definir as variáveis aleatórias de comprimento $k$ e $l: X_{i}^{(k)}=\left(X_{i}, X_{i-1}, \ldots, X_{i-k+1}\right)$ e $Y_{i}^{(l)}=\left(Y_{i}, Y_{i-1}, \ldots, Y_{i-l+1}\right)$ e, em seguida, as probabilidades de transição:

$$
p\left(x_{i+1} \mid x_{i}^{(k)}, y_{i}^{(l)}\right)=\operatorname{prob}\left(X_{i+1}=x_{i+1} \mid X_{i}^{(k)}=x_{i}^{(k)}, Y_{i}^{(l)}=y_{i}^{(l)}\right),
$$

em que $x_{i}^{(k)}=\left(x_{i}, x_{i-1}, \ldots, x_{i-k+1}\right)$ e $y_{i}^{(l)}=\left(y_{i}, y_{i-1}, \ldots, y_{i-l+1}\right)$ são os estados de $X_{i}^{(k)}$ e $Y_{i}^{(l)}$, respectivamente. A equação (1) denota a probabilidade de se encontrar $X_{i+1}$ no estado $x_{i+1}$ quando $X_{i}^{(k)}$ e $Y_{i}^{(l)}$ estão no estado $x_{i}^{(k)}$ e $y_{i}^{(l)}$, respectivamente. Se o estado futuro $x_{i+1}$ de $X_{i}$ depende dos $k$-ésimos estados $x_{i}^{(k)}$ e os $l$-ésimos estados $y_{i}^{(l)}$, a entropia de transferência TE é definida como 


$$
T E_{Y \rightarrow X}(k, l)=\sum p\left(x_{i+1}, x_{i}^{(k)}, y_{i}^{(l)}\right) \log \frac{p\left(x_{i+1} \mid x_{i}^{(k)}, y_{i}^{(l)}\right)}{p\left(x_{i+1} \mid x_{i}^{(k)}\right)} .
$$

A entropia de transferência é uma medida assimetrica $\left(T E_{Y \rightarrow X} \neq T E_{X \rightarrow Y}\right)$, gerando a Net Transfer Entropy:

$$
T E_{\text {net }}(Y \rightarrow X)=T E(Y \rightarrow X)-T E(X \rightarrow Y) .
$$

Se $T E_{n e t}(Y \rightarrow X)>0$, considera-se que o fluxo de informação acontece de $Y$ para $X$; caso contrário, de $X$ para $Y$ (He \& Shang, 2017).

\section{Resultados e Discussão}

Transfer Entropy (TE) e Net Transfer Entropy (Net TE) foram aplicadas nas séries de preços das commodities agrícolas nos períodos: pré-crise, que vai de julho de 1997 até junho de 2007, e pós-crise, de julho de 2007 a dezembro de 2019. Esses períodos foram assim defindos de acordo com a literatura (Kristoufek, Janda, \& Zilberman, 2012).

As séries analisadas são apresentadas na Figura 1, onde se pode observar que os preços de todas as commodities aumentaram depois da crise.

Figura 1. Séries temporais dos preços das commodities agrícolas: açúcar, boi gordo, café e soja. A linha vermelha separa os períodos pré-crise e pós-crise.
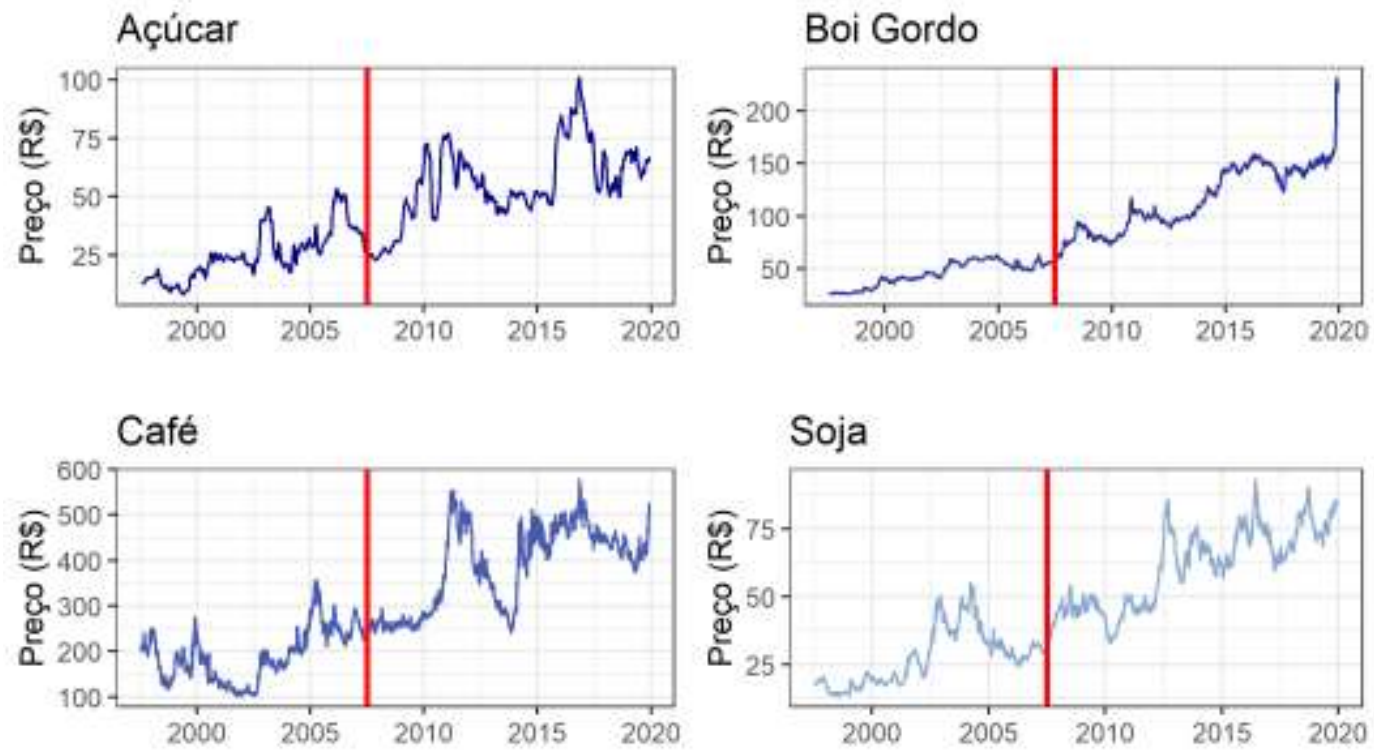

Fonte: Elaborados pelos autores a partir dos dados do CEPEA (2020).

Os valores de TE (na direção das commodities listadas na primeira coluna para as da primeira linha) para as séries de preços no período pré-crise são apresentados na Tabelas 2 e, para período pós-crise, nas Tabela 3. 
Tabela 2. TE entre as séries de preço das commodities para o período pré-crise (07/1997 - 06/2007). A direção da informação é das commodities listadas na primeira coluna para as da primeira linha.

\begin{tabular}{l|c|c|l|l}
\hline Commodity & Açúcar & Boi Gordo & Café & Soja \\
\hline Açúcar & & 0,1593 & 0,0881 & 0,1242 \\
\hline Boi Gordo & 0,1509 & & 0,0766 & 0,1265 \\
\hline Café & 0,1039 & 0,0963 & & 0,1041 \\
\hline Soja & 0,1644 & 0,1525 & 0,0673 & \\
\hline
\end{tabular}

Fonte: Autores.

Tabela 3. TE entre as séries de preço das commodities para o período pós-crise (07/2007 - 12/2019). A direção da informação é das commodities listadas na primeira coluna para as da primeira linha.

\begin{tabular}{l|c|c|l|l}
\hline Commodity & Açúcar & Boi Gordo & Café & Soja \\
\hline Açúcar & & 0,1274 & 0,0768 & 0,1568 \\
\hline Boi Gordo & 0,1340 & & 0,0906 & 0,1482 \\
\hline Café & 0,1278 & 0,1157 & & 0,1295 \\
\hline Soja & 0,1535 & 0,1416 & 0,0764 & \\
\hline
\end{tabular}

Fonte: Autores.

Subtraindo os valores de TE apresentados nas Tabelas 2 e 3, obtiveram-se os valores de Net TE para cada par de commodities, para avaliar a direção do fluxo de informação em cada um dos períodos, pré e pós-crise. Estes valores são apresentados na Tabela 4 e graficamente na Figura 2. Observa-se para as séries dos preços, que: após a crise (Figura 2), houve alteração de fluxo de informação entre açúcar e boi (de Açúcar $\rightarrow$ Boi para Boi $\rightarrow$ Açúcar), Açúcar e Soja (de Soja $\rightarrow$ Açúcar para Açúcar $\rightarrow$ Soja) e boi e soja (de Soja $\rightarrow$ Boi para Boi $\rightarrow$ Soja), enquanto a série de preços de café transmitiu informação para as das outras commodities em ambos os períodos, antes e depois da crise (Café $\rightarrow$ Açúcar, Café $\rightarrow$ Boi, Café $\rightarrow$ Soja). Nota-se também que a maior transferência de informação (maior valor de NET TE) foi da série de preços de café para os de soja no período pós crise $(\mathrm{TE}=0,0531)$, enquanto a menor transferência de informação foi da série de preços de açúcar para os de soja, também no período pós-crise (TE = 0,0033).

A transferência total (soma dos valores absolutos de Net TE para todos os pares de commodities) antes da crise foi 0,1469 e depois da crise, 0,1457 , indicando que o mercado dessas commodities foi um pouco mais eficiente depois da crise (menor valor de TE total). 
Tabela 4. Net TE entre as séries de preços das commodities para os períodos antes e depois da crise.

\begin{tabular}{c|r|r}
\hline Commodities & Pré-crise & Pós-crise \\
\hline Açúcar $\rightarrow$ Boi & 0,0084 & $-0,0066$ \\
\hline Açúcar $\rightarrow$ Café & $-0,0158$ & $-0,0510$ \\
\hline Açúcar $\rightarrow$ Soja & $-0,0402$ & 0,0033 \\
\hline Boi $\rightarrow$ Café & $-0,0197$ & $-0,0251$ \\
\hline Boi $\rightarrow$ Soja & $-0,0260$ & 0,0066 \\
\hline Café $\rightarrow$ Soja & 0,0368 & 0,0531 \\
\hline
\end{tabular}

Fonte: Autores.

Figura 2. Net TE entre as séries de preços das commodities para os períodos pré-crise (azul) e pós-crise (vermelho).

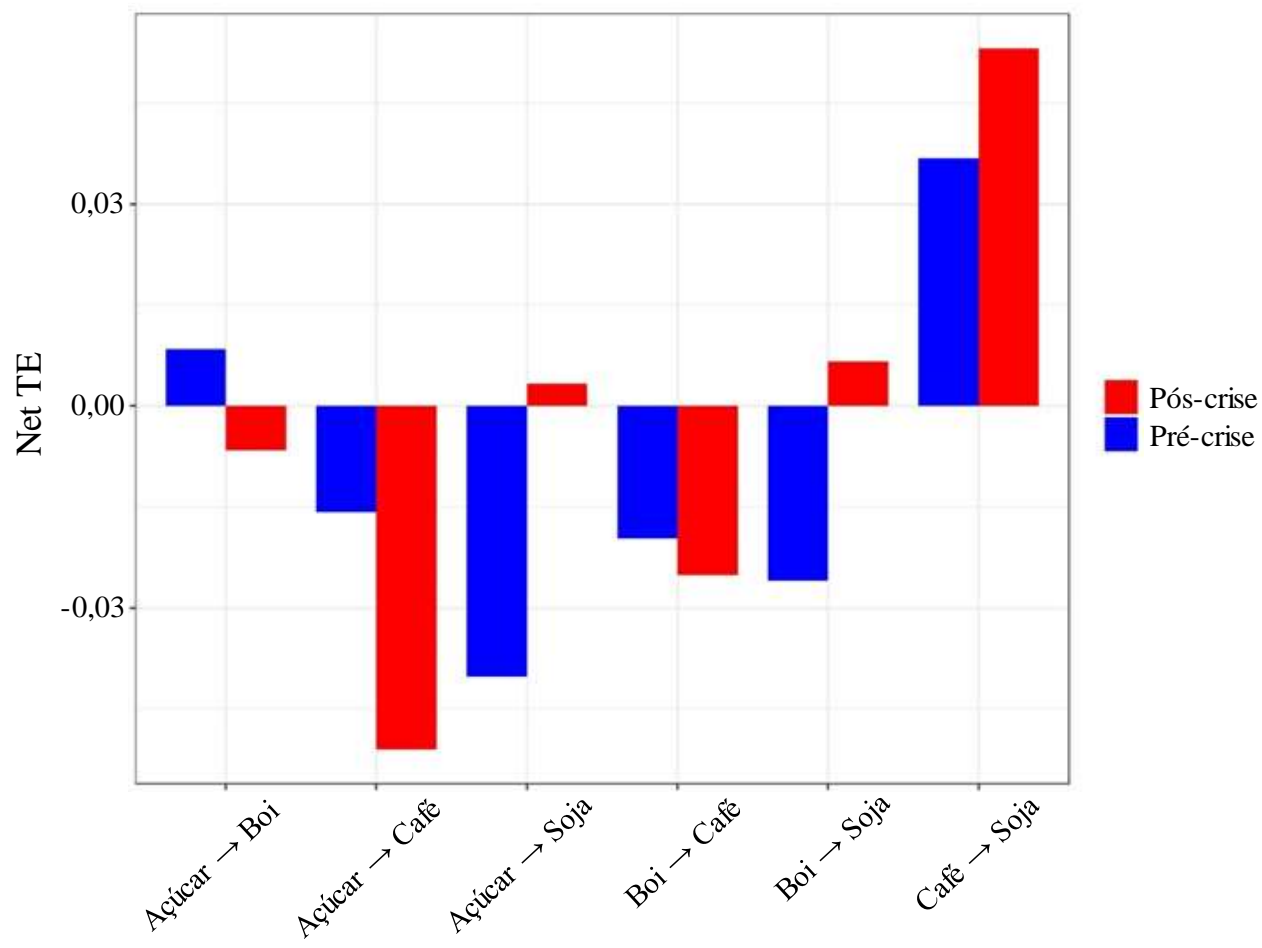

Commodities

Fonte: Autores.

A transferência de informação com uso de TE foi estudada para várias séries financeiras. Sensoy, et al. (2014) analisaram o fluxo de informações entre taxas de câmbio e preços de ações em vários países emergentes. A análise mostrou que, antes da crise de 2008, apenas existe uma interação de baixo nível entre essas duas variáveis, e as taxas de câmbio dominam os preços das ações em geral. Durante a crise, surge uma forte interação bidirecional e, no período pós-crise, a forte interação continua existindo e, em geral, os preços das ações dominam as taxas de câmbio. Gao e Mei (2019) investigaram a estrutura de correlação entre os mercados dos EUA e os mercados asiáticos durante a crise financeira de 2008 utilizando TE e encontraram uma dependência mais forte após a crise. Os dois estudos citados envolvem mercados internacionais, enquanto neste artigo foi analisado o mercado local, brasileiro, para variáveis financeiras de mesmo tipo: commodities agrícolas. Apesar 
das diferenças dos mercados estudados, os resultados aqui obtidos também mostraram que a crise de 2008 afetou a relação entre os mercados financeiros, alterando a direção de fluxo de informação entre as séries analisadas.

\section{Considerações Finais}

Os resultados da análise de Transfer Entropy mostraram que a crise de 2008 afetou a direção de transferência de informação entre os pares açúcar-boi, açúcar-soja e boi-soja, enquanto a série de preços do café transmitiu informação para outras commodities em ambos os períodos, antes e depois da crise. A maior transferência de informação foi da série de preços de café para soja no período pós-crise, enquanto a menor transferência de informação foi da série de preços de açúcar para soja, também no período pós-crise. A transferência total (soma dos valores absolutos de Net TE para todos os pares de commodities) foi pouco menor no período pós-crise, indicando que a crise afetou o mercado dessas commodities em direção da maior eficiência, já que a menor transferência entre as commodities indica menor correlação entre seus preços, característica de mercados mais eficientes.

Como trabalhos futuros, seria igualmente de interesse analisar outras commodities agrícolas do mercado brasileiro e dos mercados agrícolas internacionais, como carne suína, algodão e milho.

\section{Agradecimentos}

Os autores agradecem, pelo apoio e incentivo, à Universidade Federal Rural de Pernambuco (UFRPE), ao Programa de Pós-Graduação em Biometria e Estatística Aplicada (PPGBEA), à Coordenação de Aperfeiçoamento de Pessoal de Nível Superior (CAPES), ao Conselho Nacional de Desenvolvimento Científico e Tecnológico (CNPq), e à Fundação de Amparo à Ciência e Tecnologia do Estado de Pernambuco (FACEPE).

\section{Referências}

Alan Bojanic, H. (2017). The rapid agricultural development of Brazil in the last 20 years.

Beckmann, J., \& Czudaj, R. (2014). Volatility transmission in agricultural futures markets. Economic Modelling, 36, 541-546.

dos Santos Caliari, S. C. (2019). A Exportação De Carne Bovina No Brasil. CIMATech, 1(6), 281-292.

Farias, D. B. C., Silva, A. S. A., Stosic, T., \& Stosic, B. (2020). Análise de entropia multiescala da dinâmica de preços de commodities agrícolas brasileiras. Research, Society and Development, 9(11), e4739119832.

Gao, H. L., \& Mei, D. C. (2019). The correlation structure in the international stock markets during global financial crisis. Physica A: Statistical Mechanics and its Applications, 534, 122056.

He, J., \& Shang, P. (2017). Comparison of transfer entropy methods for financial time series. Physica A: Statistical Mechanics and its Applications, 482, 772785 .

Ivanic, M., Martin, W., \& Zaman, H. (2012). Estimating the Short-Run Poverty Impacts of the 2010-11 Surge in Food Prices. World Development, 40(11), 2302-2317.

Jale, J. S., Júnior, S. F., Stošić, T., Stošić, B., \& Ferreira, T. A. (2019). Information flow between Ibovespa and constituent companies. Physica A: Statistical Mechanics and its Applications, 516, 233-239.

Kristoufek, L., Janda, K., \& Zilberman, D. (2012). Correlations between biofuels and related commodities before and during the food crisis: A taxonomy perspective. Energy Economics, 34(5), 1380-1391.

Lima, C. R. A., de Melo, G. R., Stosic, B., \& Stosic, T. (2019). Cross-correlations between Brazilian biofuel and food market: Ethanol versus sugar. Physica A: Statistical Mechanics and its Applications, 513, 687-693.

Nazlioglu, S., \& Soytas, U. (2012). Oil price, agricultural commodity prices, and the dollar: A panel cointegration and causality analysis. Energy Economics, 34(4), 1098-1104.

Orange, N., \& Abaid, N. (2015). A transfer entropy analysis of leader-follower interactions in flying bats. The European Physical Journal Special Topics, 224(17), 3279-3293. 
Research, Society and Development, v. 10, n. 7, e2110715978, 2021

(CC BY 4.0) | ISSN 2525-3409 | DOI: http://dx.doi.org/10.33448/rsd-v10i7.15978

Pessoa, R. V. S., Barreto, I. D. de C., Araújo, L. da S., Moreira, G. R., Stosic, T., \& Stosic, B. (2021). Correlações em séries temporais de preços de frango, soja e milho. Research, Society and Development, 10(4), e20610414019.

Schreiber, T. (2000). Measuring information transfer. Physical review letters, 85(2), 461.

Sensoy, A., Sobaci, C., Sensoy, S., \& Alali, F. (2014). Effective transfer entropy approach to information flow between exchange rates and stock markets. Chaos, solitons \& fractals, 68, 180-185.

Shaffer, I., \& Abaid, N. (2020). Transfer Entropy Analysis of Interactions between Bats Using Position and Echolocation Data. Entropy, $22(10), 1176$.

Ursino, M., Ricci, G., \& Magosso, E. (2020). Transfer Entropy as a Measure of Brain Connectivity: A Critical Analysis With the Help of Neural Mass Models. Frontiers in computational neuroscience, 14, 45.

Van Milligen, B. P., Birkenmeier, G., Ramisch, M., Estrada, T., Hidalgo, C., \& Alonso, A. (2014). Causality detection and turbulence in fusion plasmas. Nuclear Fusion, 54(2), 023011.

Vicente, R., Wibral, M., Lindner, M., \& Pipa, G. (2011). Transfer entropy - a model-free measure of effective connectivity for the neurosciences. Journal of computational neuroscience, 30(1), 45-67.

Wing, S., Johnson, J. R., Camporeale, E., \& Reeves, G. D. (2016). Information theoretical approach to discovering solar wind drivers of the outer radiation belt. Journal of Geophysical Research: Space Physics, 121(10), 9378-9399. 